\title{
Review: quetiapine leads to fewer withdrawals because of adverse effects in schizophrenia
}

\author{
Srisurapanont M, Disayavanish C, Taimkaew K. Quetiapine for schizophrenia. Cochrane Review, latest version, 27 May 1998. In: \\ Cochrane Library. Oxford: Update Software.
}

\section{Question}

In patients with schizophrenia, is quetiapine effective and what are the adverse effects?

\section{Data sources}

Studies were identified by searching Biological Abstracts, CINAHL, the Cochrane Library, Cochrane Schizophrenia Group's Register, EMBASE/Excerpta Medica, Medline, PsycLIT, SIGLE, and Sociofile; handsearching 6 journals and conference proceedings; and contacting experts in the field.

\section{Study selection}

Studies were selected if they were randomised controlled trials evaluating the effectiveness of quetiapine compared with placebo or other neuroleptic drugs in patients (16-65 y of age) with schizophrenia or similar illnesses.

\section{Data extraction}

Data were extracted on study design, patient characteristics, treatment type and duration, and main outcome measures.

\section{Main results}

7 short term trials met the selection criteria. In each comparison, $>30 \%$ of each participant group were lost to follow up within 12 weeks. In the studies that compared quetiapine with placebo, the number of patients leaving the study early was lower in the quetiapine group; there was greater improvement in global state and psychotic symptoms (both positive and negative) in the quetiapine group; and there were no differences for most side effects except for dizziness, dry mouth, and sleepiness. In the 3 studies that compared quetiapine with classical antipsychotics, dropout rates in both groups were high $(56 \% v 58 \%)$ and not statistically different. No difference existed between quetiapine and classical antipsychotics in clinical response. However, the difference in dropout rates because of adverse effects was statistically significant (table). In the 3 studies that compared high dose quetiapine with low dose quetiapine, fewer patients in the high dose group left the study early and a greater number improved in terms of global state.

\section{Conclusions}

Quetiapine may be more effective than placebo, and high dose quetiapine may be more effective than low dose quetiapine. No difference was found between quetiapine and some traditional antipsychotics in positive and negative symptoms. Quetiapine, however, leads to fewer withdrawals because of adverse effects when compared with some classical antipsychotics.

Source of funding: Cochrane Schizophrenia Group General Fund UK.

For correspondence:Dr M Srisurapanont, Department of Psychiatry, Chiang Mai University, PO Box 102, Amphur Muang, Chiang Mai 50202, Thailand. Fax +66 52217144

Quetiapine v some classical antipsychotics in patients with schizophrenia*

\begin{tabular}{|c|c|c|c|c|}
\hline \multirow[b]{2}{*}{ Outcome } & \multicolumn{2}{|c|}{ Weighted event rates } & \multirow[b]{2}{*}{$R R R(95 \% C I)$} & \multirow[b]{2}{*}{$N N T(C I)$} \\
\hline & Quetiapine & Antipsychotics (hal,clpz) & & \\
\hline \multirow{3}{*}{$\begin{array}{l}\text { Leaving the study early due to adverse effects } \uparrow \\
\text { Dystonia }\end{array}$} & $7 \%$ & $17 \%$ & $75 \%$ (46 to 88$)$ & 11 (6 to 42$)$ \\
\hline & $1 \%$ & $5 \%$ & $85 \%$ (21 to 97$)$ & 29 (13 to 111$)$ \\
\hline & Quetiapine & Antipsychotics (hal, clpz) & $R R I(C I)$ & $N N H(C I)$ \\
\hline Dry mouth & $10 \%$ & $3 \%$ & $185 \%$ (46 to 457$)$ & $16(10$ to 38$)$ \\
\hline Sleepiness & $16 \%$ & $11 \%$ & $51 \%(5$ to 116$)$ & 21 (11 to 188$)$ \\
\hline
\end{tabular}

*Hal $=$ haloperidol; $\mathrm{Clpz}=$ chlorpromazine. Other abbreviations defined in glossary. RRR, RRI, NNT, NNH, and CI calculated from data in article. $†$ Random effects model (statistical heterogeneity). \#Fixed effects model.

\section{Commentary}

In recent years, new antipsychotic drugs have been introduced for the treatment of schizophrenia. Srisurapanont et al evaluate the effectiveness of quetiapine in comparison with placebo and traditional antipsychotics.

The small number of patients enrolled in each comparative study represents the major problem in the evaluation of quetiapine. The lack of difference in dropouts due to treatment failure, which is the primary endpoint, is difficult to interpret. In fact, the number of patients analysed is not large enough to detect any real difference; equivalence of effect therefore cannot be claimed. The only conclusion is that at present quetiapine cannot be considered more effective than traditional antipsychotics. Another problem is that clinical trials compare the mean change from baseline in the active and control group, but rarely do these studies provide the number of patients in each group showing a certain reduction in the score. This information would have been of value to provide a better idea of the clinical benefit of the new compound. ${ }^{1}$

Srisurapanont et al found that quetiapine leads to fewer withdrawals because of extrapyramidal symptoms (EPSs) in comparison with traditional antipsychotics. However, they suggest caution because half of the patient data were missing. It should also be noted that more than two thirds of patients receiving the control treatment were treated with haloperidol. Among tra- ditional antipsychotics, haloperidol has the worst profile for EPSs, and therefore it cannot be considered the gold standard with reference to this outcome measure.

The available data suggest that quetiapine should not be used in everyday clinical practice because we lack evidence of superiority of effect over traditional drugs, equivalence of effect is far from being assessed, and the safe extrapyramidal profile has to be confirmed in comparison with other traditional antipsychotics and in the long term.

Corrado Barbui, MD Istituto di Ricerche Farmacologiche "Mario Negri" Milan, Italy

1 Barbui C, Garattini S. Int Clin Psychopharmacol 1999;14:133-7. 